\title{
28 Research Suare \\ Eye Disorders Spectrum: A Tertiary Hospital Pediatric Ophthalmology Clinic Based in Ethiopia
}

\section{Yohannes Tewolde Kidane}

Addis Ababa University

Addisu Worku Teshome ( $\square$ addiaddu@yahoo.com )

Addis Ababa University

\section{Research Article}

Keywords: Eye disorders, pediatric eye clinic, spectrum, tertiary hospital

Posted Date: November 15th, 2021

DOI: https://doi.org/10.21203/rs.3.rs-1044839/v1

License: (c) (1) This work is licensed under a Creative Commons Attribution 4.0 International License.

Read Full License

Version of Record: A version of this preprint was published at BMC Ophthalmology on March 12th, 2022. See the published version at https://doi.org/10.1186/s12886-022-02336-8. 


\section{Abstract}

Background: Epidemiological studies to determine the pattern of eye disorders among children are important for proper health care planning and management. This study aimed to document the spectrum and frequency of eye diseases of children who attended the pediatric ophthalmology clinic of a tertiary teaching hospital Addis Ababa, Ethiopia.

Methods: A cross sectional and convenient sample of 1237, male and female children (16 years and below) with ocular disorders presenting for the first time and those children with settled diagnosis coming for follow up visit between June 1, 2018 to May 31, 2019 were included in the study. Data on age at presentation, sex and diagnosis were collected and analyzed. Eye disorders were classified into various categories. Children were grouped into four age groups. Ratios, percentages and chi square associations were calculated. $P<0.05$ was considered statistically significant.

Results: Of the children 60\% were male. The mean age (standard deviation) of the children was 4.26 $\pm 4.1)$ years. Patients aged $0-5$ years old were the largest group constituting $70.5 \%$. Ocular motility imbalances were the most common ocular disorders seen (32.8\%), followed by childhood cataract (18.4\%) and infection and inflammation of eye and adnexa (8.3\%). Ocular motility imbalances were recorded more frequently and statistically significant $(p<0.001)$ among 15 years age group. Within the childhood cataract category, congenital cataract was more prominent (7.1\%). Within the infection and inflammation category, cornea/ sclera infections were more common (3.7\%).

Conclusions: The study highlights common eye disorders seen in children in a specialized hospital ophthalmic clinic. Ocular motility imbalance, childhood cataract, and infection and inflammation of the eye and adnexa, were the most common occurring disorders. Early presentation was common, and males were more affected than females.

\section{Introduction}

Pediatric Eye Disorders pattern varies widely across the globe, being largely determined by socioeconomic development, and the availability of primary health care and eye care services (1). In high income countries, lesions of the optic nerve and higher visual pathways predominate as the cause of blindness, while corneal scarring from measles, vitamin A deficiency, the use of harmful traditional eye remedies, and swelling of the child's eyelids due to infection during birth are the major causes of blindness in developing countries. Retinopathy of prematurity is an important cause in middle income countries (2). Other significant causes in all countries are congenital abnormalities, such as cataract, glaucoma, and hereditary retinal dystrophies (1).

Worldwide prevalence of blindness is $0.78 / 1000$ and there are estimated 1.4 million blind children, three fourth of whom live in developing countries in Africa and Asia (3). In Ethiopia the prevalence of childhood blindness was $0.1 \%$ accounting for $6 \%$ of overall blindness (4). 
There is a lack of accurate and reliable data on the pattern of pediatric eye disorders at Menelik II Hospital a tertiary pediatric eye care center. Description of the pattern of eye disorders among children is important since prevention and treatment of childhood blindness is disease specific (1). Few hospital based studies are available on pattern of pediatric eye disorders in Ethiopia $(5,6)$. Given the variation in study designs, sample sizes, the effects of different geographical and socioeconomic situations, and relative importance can change over time, we wanted to document the pattern of eye disorders in a pediatric population seen at Menelik II Hospital pediatric ophthalmology clinic in Addis Ababa, Ethiopia over one year period and with greatest participant numbers than previously.

The findings obtained from this hospital based study needed for planning and evaluating preventive and curative services for children to reduce sever visual impairment and blindness.

\section{Patients And Methods}

A hospital based prospective cross-sectional study was conducted at Menelik II Hospital from June 1, 2018 to May 31, 2019. Menelik II Hospital is a tertiary eye care teaching hospital located in the capital city Addis Ababa, and serves as a referral center for all the primary, secondary and tertiary eye health care facilities from all parts of the country. It is the first tertiary and ophthalmology residency teaching hospital, and has subspecialists and general ophthalmologists in the different subspecialty units of Ophthalmology Department. The pediatric ophthalmology clinic is manned by consultant pediatric ophthalmologists who supervise the residents posted to the unit. It runs 5 days in a week. Every new patient attending the clinic is consulted and reviewed by the pediatric ophthalmologist who subjects the patients to examination and relevant investigations before a definitive diagnosis is made.

During the study period authors invited all consecutive children under 16 years of age with eye disorder presenting for the first time and those children with settled clinical diagnosis coming for follow up at the hospital pediatric ophthalmology clinic and seen by the consultant pediatric ophthalmologist.

Ocular examination was performed starting with visual acuity using different methods for age. Fixation and the ability to follow light in infants, colorful fixation target and CSM (central, steady and maintained) methods were used to assess visual acuity in 14 years old children. For children 4 years and above Snellen's visual acuity chart was used. Hirschberg and cover uncover tests were also carried out to diagnose strabismus and latent squint. Examination of the eyelid margins, conjunctivae, the cornea and anterior segment of the eye was performed with the aid of a slit lamp, pen torch light and a magnifying loupe. Intraocular pressure measurement was done using Perkin handheld tonometer in suspected cases of childhood glaucoma. A direct ophthalmoscope, $90 \mathrm{D}$ Volk, and both were used to examine the posterior segment after dilatation of both pupils using 1\% mydriacyl. Refraction was performed routinely under cycloplegia. Ptosis evaluation included vertical fissure height, lid margin reflex distance, lid to crease distance and levator function test using ruler. Laboratory investigations and imaginings were done whenever required to elicit diagnosis. Consultations to other ophthalmic subspecialists, pediatricians and 
other discipline specialists were made when necessary. An examination under anesthesia was carried out to confirm a diagnosis when required.

At the end of the consultation, one or more diagnoses were recorded in each patient's medical chart. One main diagnosis which represented the disorder that was primarily responsible for the outpatient services provided was reported for each patient in the study. In those children with settled clinical diagnosis coming for follow up visit during the study period the initial diagnosis was accepted as disorder. Any new disorder arising during hospital subsequent visit was ignored.

Ethical approval and permission to conduct the study was obtained from ethical review committee of Department of Ophthalmology, School of Medicine, Addis Ababa University and was carried out in accordance with the tenets of the declaration of Helsinki. Parent, guardian, or both were informed of the purpose of the study and had to give their oral informed consent before the child being enrolled.

Data were collected by the author (Y.T.K) among children who attended the eye clinic using structured questionnaire. Age at presentation, sex, and clinical diagnosis were extracted on a daily base.

The disorders were grouped into 14 categories; any disorder which did not fit into these categories was included in other categories. The disorder categories included were ocular motility imbalance, childhood cataract, congenital anomalies, infection/ inflammation, orbital/ ocular tumors, orbital, ocular and both trauma, childhood glaucoma, refractive error, nasolacrimal duct obstruction, ocular allergies, ptosis, cortical visual impairment/ blindness, retinopathy of prematurity and ectopia lentis.

Patients were grouped by age into infants (01 year), toddlers and preschool (2-5years), school age (610 years) or older children and teenagers (11-16years). Data entry in the computer was done by excel spread sheet and data analysis was done using SPSS version 20 software. The Chi square test was used to compare variables and a $p$ value less than 0.05 was considered statistically significant. Ratios and percentages were calculated and tabulated. Possible associations with age groups and sex, age groups and ocular disorders, and sex and ocular disorders were pursued using $p$ values. The results were described, summarized and presented in figure and tables.

\section{Results}

A total of 1326 new and follow up pediatric patients who were seen in pediatric ophthalmic clinic at Menelik II hospital during the study period, who met the inclusion criteria, were recruited for this study. As incomplete questionnaires were discarded, data obtained from 1237 respondents were analyzed, making the response rate $93.3 \%$. The mean $( \pm S D)$ age of the pediatric patients evaluated in pediatric ophthalmic clinic were $4.3( \pm 4.1)$ years. Their age ranged between 5 days and 15 years. There were $692(60 \%)$ males and 545 (40\%) females. Figure 1 shows the age group and sex distribution of the children seen, with a male preponderance in all age group. Fig. 1 also shows the highest frequency of consultation was recorded among pediatric patients up to 5 years, constituting $70.5 \%$ of the patients (Chi square, $p=0.272$ ), with a maximum number of patients (47.4\%) presented between 1-and 5-years age group. 
Table 1 shows the spectrum and frequency of various pediatric eye disorders seen. Eye motility disorders were the most common disorders seen (32.8\%). This was followed by childhood cataract (18.4\%), infection/ inflammation (8.3\%), orbital/ ocular tumors (7.1\%) and congenital anomalies (6.8\%). The most common form of ocular motility imbalance was convergent strabismus (18.8\%).

Blunt ocular trauma (1.6\%) was the most common form of orbital and ocular trauma seen followed by penetrating injuries (1.4\%) and superficial foreign body (0.6\%). Under the category of others included pseudo strabismus $(0.16 \%)$, conjunctiva nevus $(0.48 \%)$ and coat's disease $(0.16 \%)$.

Table 1. Spectrum of pediatric eye disorders as a percentage of total sample at pediatric ophthalmic clinic, Menelik II hospital, Addis Ababa, 2019, N=1237. 


\begin{tabular}{|c|c|c|}
\hline Ocular disorders & number & $\%$ \\
\hline Ocular motility imbalance & 406 & 32.8 \\
\hline Convergent strabismus & 232 & 18.8 \\
\hline Divergent strabismus & 110 & 8.9 \\
\hline Vertical strabismus & 10 & 0.8 \\
\hline Complex strabismus & 19 & 1.5 \\
\hline Nystagmus & 23 & 1.9 \\
\hline Amblyopia & 12 & 1.0 \\
\hline Childhood cataract & 227 & 18.4 \\
\hline Congenital cataract & 88 & 7.1 \\
\hline Traumatic cataract & 76 & 6.1 \\
\hline Developmental cataract & 63 & 5.1 \\
\hline Infection/ inflammation & 103 & 8.3 \\
\hline Lid (Stye/ chalazion/ blepharitis) & 14 & 1.1 \\
\hline Conjunctiva (viral/ bacteria) & 17 & 1.4 \\
\hline Cornea/ sclera & 46 & 3.7 \\
\hline Uvea & 11 & 0.9 \\
\hline Vitreous/ retina & 6 & 0.5 \\
\hline Optic nerve & 9 & 0.7 \\
\hline Orbital/ ocular tumor & 88 & 7.1 \\
\hline Retinoblastoma & 66 & 5.3 \\
\hline Rabdomyosarcoma & 10 & 0.8 \\
\hline Capillary hemangioma & 6 & 0.5 \\
\hline Optic nerve glioma & 6 & 0.5 \\
\hline Congenital anomalies & 84 & 6.8 \\
\hline Congenital entropion/ ectropion/ epiblepharon & 20 & 1.6 \\
\hline Dermoid cyst & 18 & 1.5 \\
\hline Microphthalmia & 14 & 1.1 \\
\hline
\end{tabular}




\begin{tabular}{|c|c|c|}
\hline CHED & 5 & 0.4 \\
\hline Coloboma & 11 & 0.9 \\
\hline PFV & 9 & 0.7 \\
\hline RP & 3 & 0.2 \\
\hline Congenital CN palsy (CN III/ IV/ VI) & 6 & 0.5 \\
\hline Childhood glaucoma & 76 & 6.1 \\
\hline Congenital glaucoma & 55 & 4.4 \\
\hline Juvenile open angle glaucoma & 10 & 0.8 \\
\hline Secondary glaucoma & 11 & 0.9 \\
\hline Refractive error & 64 & 5.2 \\
\hline Myopia & 34 & 2.7 \\
\hline Hyperopia & 23 & 1.9 \\
\hline Astigmatism & 7 & 0.6 \\
\hline Ocular/ orbital trauma & 52 & 4.2 \\
\hline Blunt trauma & 20 & 1.6 \\
\hline Penetrating injury & 17 & 1.4 \\
\hline Non penetrating injury & 8 & 0.6 \\
\hline Superficial foreign body (Conjunctiva/ cornea) & 8 & 0.6 \\
\hline Ocular allergies & 36 & 2.9 \\
\hline Vernal keratoconjunctivitis & 22 & 1.8 \\
\hline Other allergies & 14 & 1.1 \\
\hline Congenital Nasolacrimal duct obstruction & 33 & 2.7 \\
\hline Ptosis & 25 & 2.0 \\
\hline Cortical visual impairment/ blindness & 17 & 1.4 \\
\hline Retinopathy of prematurity & 10 & 0.8 \\
\hline Ectopia lentis & 6 & 0.5 \\
\hline Others & 10 & 0.8 \\
\hline Total & 1237 & 100 \\
\hline
\end{tabular}


The spectrum and frequency of eye diseases varied across the four age groups (Table 2). The difference in presentation by age group was statistically significant among children with different eye diseases. The ocular motility imbalance was predominant (58.4\%) among 15 years age group (Chi square, $p<0.001$ ). Childhood cataracts were the most prevalent disorder (35.7\%) affecting age group 510 years (Chi square, $p<0.001)$. The prevalence of congenital anomalies (81.0\%) was significantly higher in children under 5 years (Chi square, $p<0.001$ ).

Table 2. Distribution of pediatric eye disorders across age groups among children attending pediatric eye clinic at Menelik II Hospital, Addis Ababa, 2019 ( $N=1237)$. 


\begin{tabular}{|c|c|c|c|c|c|}
\hline & $<1 \mathrm{yr}$ & $1-5 y r s$ & $6-10 y r s$ & $11-15 y r s$ & Total \\
\hline & $(\%)$ & $(\%)$ & $(\%)$ & $(\%)$ & $(\%)$ \\
\hline Ocular motility imbalance & $49(12.1)$ & $237(58.4)$ & $59(14.5)$ & $61(15.0)$ & $406(32.8)$ \\
\hline Childhood cataract & $48(21.1)$ & $81(35.7)$ & $63(27.8)$ & $35(15.4)$ & $227(18.4)$ \\
\hline Infection/ inflammation & $23(22.3)$ & $50(48.5)$ & $23(22.3)$ & $7(6.8)$ & $103(8.3)$ \\
\hline Orbital/ ocular tumor & $14(15.9)$ & $60(68.2)$ & $8(9.1)$ & $6(6.8)$ & $88(7.1)$ \\
\hline Congenital anomalies & $42(50.0)$ & $26(31.0)$ & $12(14.3)$ & $4(4.8)$ & $84(6.8)$ \\
\hline Childhood glaucoma & $43(56.6)$ & $20(26.3)$ & $9(11.8)$ & $4(5.3)$ & $76(6.1)$ \\
\hline Refractive error & $7(10.9)$ & $32(50.0)$ & $18(28.1)$ & $7(10.9)$ & $64(5.2)$ \\
\hline Orbital/ ocular trauma & $7(13.5)$ & $22(42.3)$ & $18(34.6)$ & $5(9.6)$ & $52(4.2)$ \\
\hline Ocular allergies & $3(8.3)$ & $11(30.6)$ & $13(36.1)$ & $9(25.0)$ & $36(2.9)$ \\
\hline Congenital nasolacrimal & $12(36.4)$ & $21(63.6)$ & - & - & $33(2.7)$ \\
\hline
\end{tabular}

duct obstruction

Ptosis

$10(40.0 \%) \quad 14(56.0)$

1(4.0)

$25(2.0)$ 
$-$

$10(0.8)$

Ectopia lentis

$\begin{array}{ll}- & 4(66.7)\end{array}$

2(33.3)

$6(0.5)$

Others

4(40.0)

$5(50.0)$

1(10.0)

10(0.8)

Total

\section{6(23.1)}

\section{6(45.4)}

226(18.3)

139(11.2)

This study showed that there were statistically significant differences in eye disorders in male and female children (Chi square, $p=0.015$ ). (Table 3)

Table 3. Sex distribution of eye disorders in children attending pediatric eye clinic at Menelik II Hospital, Addis Ababa, 2019 ( $\mathrm{N}=1237)$. 


\begin{tabular}{|c|c|c|c|}
\hline & Male (\%) & Female (\%) & Total (\%) \\
\hline Ocular motility imbalance & $222(54.7)$ & $184(45.3)$ & 406(32.8) \\
\hline Childhood cataract & $139(61.2)$ & $88(38.8)$ & $227(18.4)$ \\
\hline Infection/ inflammation & $55(53.4)$ & $48(46.6)$ & 103(8.3) \\
\hline Orbital/ ocular tumor & $48(54.5)$ & $40(45.5)$ & $88(7.1)$ \\
\hline Congenital anomalies & $36(42.9)$ & $48(57.1)$ & $84(6.8)$ \\
\hline Childhood glaucoma & $51(67.1)$ & $25(32.9)$ & $76(6.1)$ \\
\hline Refractive error & $30(46.9)$ & $34(53.1)$ & $64(5.2)$ \\
\hline Orbital/ ocular trauma & $38(73.1)$ & $14(26.9)$ & $52(4.2)$ \\
\hline Ocular allergies & $23(63.9)$ & 13(36.1) & $36(2.9)$ \\
\hline Congenital Nasolacrimal duct obstruction & $15(45.5)$ & $18(54.5)$ & $33(2.7)$ \\
\hline Ptosis & $12(48.0)$ & $13(52.0)$ & $25(2.0)$ \\
\hline Cortical visual impairment/ blindness & $7(41.2)$ & $10(58.8)$ & $17(1.4)$ \\
\hline Retinopathy of prematurity & $6(60.0)$ & $4(40.0)$ & $10(0.8)$ \\
\hline Ectopia lentis & $5(83.3)$ & $1(16.7)$ & $6(0.5)$ \\
\hline Others & $\begin{array}{l}5(50.0) \\
\text { ge } 11 / 17\end{array}$ & $5(50.0)$ & $10(0.8)$ \\
\hline
\end{tabular}




\section{Discussion}

The results of this study provide supporting evidence that pediatric eye disorders vary from one country to another, one region to another, and even one society to another. Therefore, there is a continuing need for further studies to provide up to date information on the trend of the problems. Ethiopia lacks robust information about the spectrum and frequency of pediatric eye disorders, although this information is essential for planning and evaluation of preventive and curative services for children with ocular morbidity. Spectrum and frequency of pediatric eye disorders in the present study was different from the previous studies conducted elsewhere in the country. (5 and 6)

In the present study, male children attended pediatric eye clinic more frequently. This finding is consistent with Sethi S et al's work, that deals with pattern of common eye diseases in children at eye department of teaching hospital (7).

Studies around the world including few from Ethiopia have claimed that older age group children were seen predominantly due to chronic recurrent diseases and also the child can report any ocular symptoms to their parents or care givers (5, 8 and 9). In contrast, this study indicated that the higher frequency of the cases seen in the younger age group of less than 5 years. The early presentation of the children for ophthalmic consultation may be due to the pattern of the eye disorders those were found in this study could be detected easily by parents or care givers.

The present study has shown ocular motility disorders were the most common disorder seen in the study population. Whereas a similar type of study done by Demissie B et al. in the south western Ethiopia showed ocular surface and eyelid infections as the commonest childhood ocular diseases indicating the change in the pattern of pediatric eye disorders within the country according to the difference in population studied (6).

Cataracts are likely to be the leading cause of blindness in children in West Africa (11). Childhood cataract was seen in $18.4 \%$ of children in this study. Keeping in mind the patients were filtered out before they reach the pediatric eye clinic. This finding is comparable with a study done by CE Gilbert et al. (11).

Infection and inflammation of the eye surfaces and adnexa were seen in this study representing $8.3 \%$ of cases. This pattern of results is consistent with (7.98\%) Achigbu et al's study (12).

The prevalence of tumor in this study was $7.1 \%$ with retinoblastoma accounting for $5.3 \%$ and Rabdomyosarcoma $0.8 \%$. Retinoblastoma is the most common intraocular tumor in childhood. These trends are in line with previous results (13).

Congenital ocular anomalies were statistically associated with and occurring in preschool age as this agrees with other hospital based studies (14 and 15). 
We found much higher value for childhood glaucoma with respect to those reported by the study carried out at Tikrit teaching hospital (9), Nigeria study (15) and Pakistan study (7). This may have occurred because we had referred patients across the country for surgical attention.

Our findings confirm the prevalence of refractive error is in good agreement with a study done by Fasih $U$ et al. (13). As might have been expected, our findings were often contradictory to hypermetropia was more common during childhood (5). The difference obtained could be attributable to the age of the children and prolonged use of near vision. In this study most of the myopic children were in school age.

Although our results defer considerably from that of Onakpoya et al (14), it can nevertheless be argued that pediatric eye trauma is managed by different duty ophthalmologists assigned for evaluation and repairing any laceration under general anesthesia and the children will continue their follow up with the respective ophthalmologist at different ophthalmic clinics in the hospital.

Waddell KM (10) and Okoye $O$ et al (17) noted that higher prevalence of ocular allergies. Our results don't support their observation, in fact the responsible factors for the difference may be ethnic groups, referral system and geographical zones.

Congenital Nasolacrimal duct obstruction was found in $2.7 \%$ of children in our study. Similar report was noted in a study from Pakistan (4.07\%) (13).

Results of the current study showed that ptosis had a comparable prevalence with other clinic-based study (8).

Our findings would seem to imply that cortical visual impairments and retinopathy of prematurity are the immerging pediatric eye disorders in contrast with what was previously reported in Ethiopia (5 and 6). The reason could be increased survival rates of premature and term neonates in the country.

The strengths of this study include the large sample size, the prospective nature of the study and standardization of diagnoses selection, given that they were made by a limited number of pediatric ophthalmologists. However, it was hospital based and, as such, is not generalizable to the community. As well, milder eye conditions are usually treated in primary and secondary care centers and the profile of diagnoses in the tertiary referral hospital would not reflect them. The diagnoses mostly were clinical.

\section{Conclusion}

The most common causes of ocular disorders among children attending the tertiary pediatric eye clinic at Menelik II Hospital were ocular motility imbalance, childhood cataract, and infection and inflammation of the eye and adnexa. Early presentation was common, and males were more affected than females.

\section{Recommendation}


These ocular disorders are either preventable or treatable. Increasing community awareness, early identification and referral, and prompt treatment may lead to substantial reduction of the effect of the problem. The findings obtained from this hospital based study might be useful in improving the existing tertiary eye care facilities in order to reduce the prevalence of childhood sever visual impairment and blindness.

\section{Abbreviations}

CHED: Congenital Hereditary Eye Disease

CN: Cranial Nerve

CSM: Central, Steady and Maintained

D: Diopter

N: Number

PFV: Persistent Fetal Vasculature

RP: Retinitis Pigmentosa

SPSS: Statistical Package for Social Sciences

yrs: years

\section{Declarations}

\section{Acknowledgments}

The authors wish to express our heartfelt and sincere gratitude to Professor Abebe Bejiga, Dr Sadik Taju, and Dr Zelalem Gizachew for a watchful eye of guidance since the start to the completion of this work. Their guidance, comments, critiques, encouragements and patience has made this work possible. The authors thank Dr Sirjana Adhikari from Tilganga Institute of Ophthalmology for her critical review of the manuscript for intellectual content. We would like to thank Sr Marta Hailemariam for her tireless help in taking visual acuity in children.

\section{Author Contributions Statement}

Y.T.K and A.W.T conceived and planned the research study: Y.T.K wrote the manuscript with support from A.W.T: Y.T.K carried out data collection at the research area and analyzed, interpreted and produced the preliminary report: A.W.T helped by supervising the project, review of the manuscript, and worked on approval of the version for publication: A.W.T agreed to be accountable for all aspect of the work, 
ensuring that questions related to the accuracy or integrity of any part of the work are appropriately investigated and resolved: Both authors read and approved to the final version of the manuscript.

\section{Funding}

This research received no specific grant from any funding agency in the public, commercial or not-forprofit sectors.

\section{Availability of data and materials}

The datasets used and analyzed during the current study are available from the corresponding author on reasonable request.

\section{Ethics approval and consent to participate}

Ethical approval and permission to conduct the study was obtained from ethical review committee of Department of Ophthalmology, School of Medicine, Addis Ababa University and was carried out in accordance with the tenets of the declaration of Helsinki. Parent, guardian, or both were informed of the purpose of the study and had to give their oral informed consent before the child being enrolled.

\section{Consent for publication}

Not applicable

\section{Competing of Interests}

The authors declare that they have no conflicts of interest.

\section{References}

1. WHO fact sheets No 282. Visual Impairment and

Blindness. http://www.who.int/mediacentre/factsheets/fs282/e n/.

2. Gilbert CE et al. Prevalence of blindness and visual impairment in children - a review of available data. Ophthalmic Epidemiology, 1999, 6: 73 - 81.

3. Gilbert C and Foster A. Childhood blindness in the context of VISION 2020-the right to sight. Bull World Health Organ, 2001; 79(3): 22732.

4. Berhan S, Anthony W. Magnitude \& causes of childhood blindness \& severe visual impairment in Sekoru District, Southwest Ethiopia: a survey using the key informant method. TransR SocTrop Med Hyg, 105 (2011) 507- 511.

5. Mehari ZA. Pattern of childhood ocular morbidity in rural eye hospital, Central Ethiopia. BMC Ophthalmol 2014; 14:50 
6. Demissie BS, Demissie ES. Patterns of eye diseases in children visiting a tertiary teaching hospital: Southwestern Ethiopia. Ethiop J Health Sci 2014; 24:6974.

7. Sethi S, Sethi M J, Iqbal $R$, Khan T. Pattern of common eye diseases in children attending outpatient eye department, Khyber Teaching hospital, Peshawar. J Med Sci. 2008; 16: 99101.

8. Jose R, Sachdeva S. School eye screening and the National Program for Control of Blindness. Indian Pediatr 2009; 46:205-8.

9. Salman MS. Pediatric eye diseases among children attending outpatient eye department of Tikrit teaching hospital. Tikrit J Pharm Sci 2010; 7:95-103.

10. Waddell K M. Eliminating global avoidable blindness. J R Coll Physicians Lond. 1999; 33(6) 568-573.

11. Gilbert, C., Canovas, R., Hagan,M. et al. Causes of childhood blindness: results from West Africa, South India and Chile. Eye 7, 184 - 188 (1993).

12. Achigbu EO, Oguego NC, Achigbu K. Spectrum of eye disorders seen in a pediatric eye clinicsouth East Nigeria.Niger J Surg 2017;23:1259.

13. Fasih U, Rahman A, Shaikh A, Fahmi MS, Rais M. Pattern of Common Paediatric Diseases at Spencer Eye Hospital. Pak J Ophthalmol. 2014; 30: 104.

14. Onakpoya $\mathrm{OH}$, Adeoye AO. Eye disorders seen in a pediatric eye clinic: South East Nigeria, a Tertiary hospital study. Clinics (Sao Paulo).2009; 64: 947-52.

15. Ajaiyeoba Al, Isawumi MA, Adeoye AO, OluleyeTS. Prevalence and causes of eye diseases amongst students in South western Nigeria. Ann Afr Med 2006; 5:197 203.

16. Ayanniyi A, Mahmoud AO, Olatunji FO. Causes and prevalence of ocular morbidity among primary school children in Ilorin, Nigeria. Niger J ClinPract 2010; 13:248 53.

17. Okoye O, Umeh RE, Ezepue FU. Prevalence of eye diseases among school children in a rural south eastern Nigerian community. Rural Remote Health 2013; 13:2357.

\section{Figures}




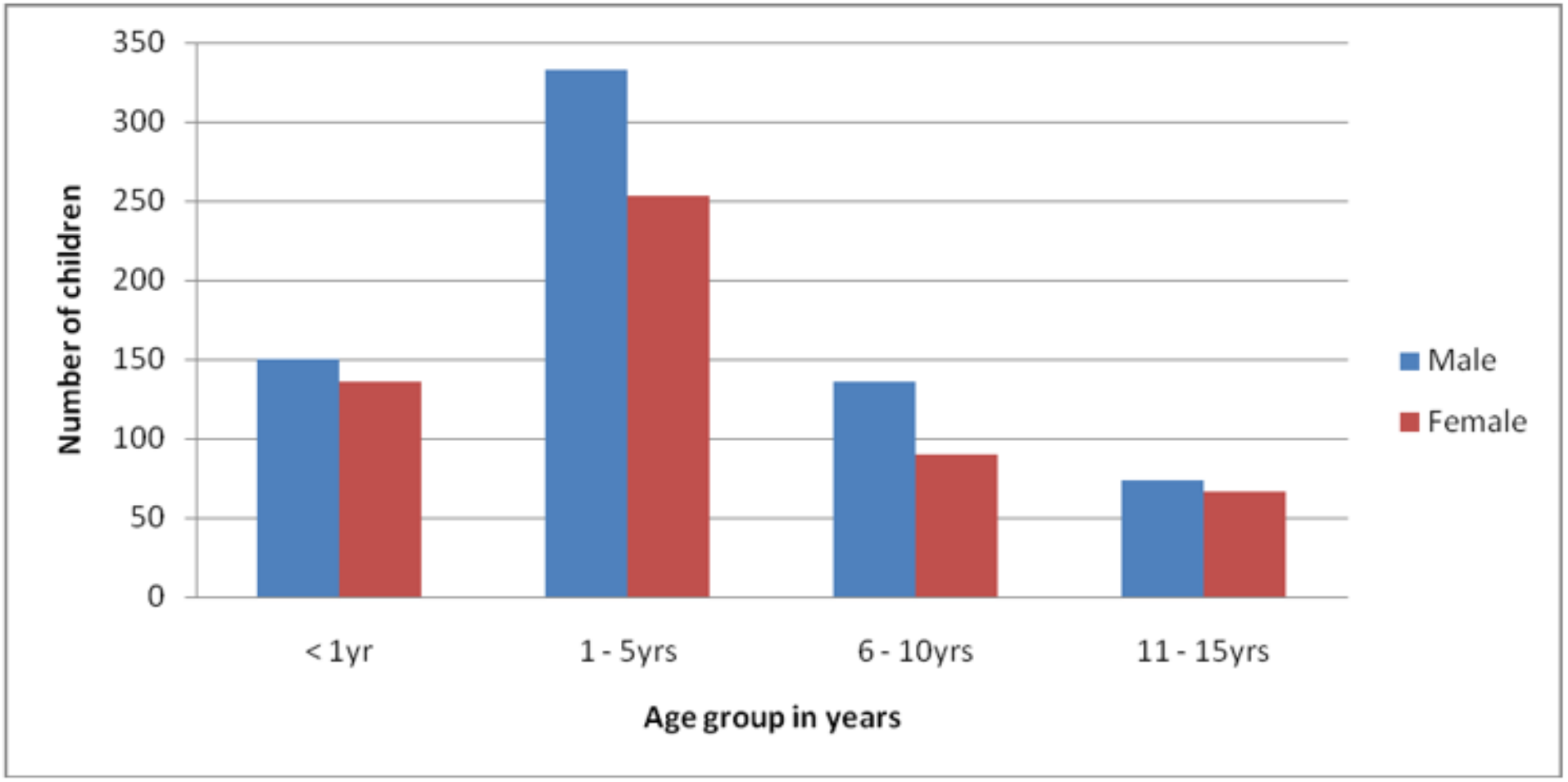

\section{Figure 1}

Age group and sex distribution of children seen at pediatric ophthalmic clinic, Menelik II hospital, Addis Ababa, 2019, N=1237. 